\title{
Determinants of diagnostic delay in chronic thromboembolic pulmonary hypertension: results from the European CTEPH Registry
}

To the Editor:

Chronic thromboembolic pulmonary hypertension (CTEPH) is characterised by chronic thrombi in the pulmonary arterial bed, causing pulmonary hypertension [1-3]. CTEPH is diagnosed in $\sim 3 \%$ of patients who survive a symptomatic acute pulmonary embolism (PE) [4]. While the surgical removal of chronic fibrotic thrombotic vascular occlusions by pulmonary endarterectomy (PEA) may cure most patients with CTEPH by normalising pulmonary artery haemodynamics and improving symptoms, patients who do not undergo the operation or do not undergo balloon pulmonary angioplasty have severe functional limitations, and poor quality of life and survival $[5,6]$. Since the natural course of CTEPH involves progressive remodelling of the distal arteries and increase of pulmonary vascular resistance, which are both important determinants of outcome, early CTEPH diagnosis and referral to an expert centre are both critical for optimal treatment. It has, however, been shown that early diagnosis of CTEPH remains a major challenge, as demonstrated by a median diagnostic delay of 14 months identified in the large European CTEPH Registry [7]. The most likely explanations for this are diagnostic misclassifications as acute PE or other conditions, the nonspecific and often insidious clinical presentation of CTEPH, and the cumbersome diagnostic process of CTEPH, which involves multiple healthcare providers from different clinical specialties $[8,9]$. The main determinants for and consequences of the diagnostic delay in CTEPH have not been studied.

This was an analysis of the prospective European CTEPH registry that included patients with CTEPH who did not receive PAH-targeted treatment before diagnosis from CTEPH expert centres in Europe and Canada between 2007 and 2009. Details of the registry have been presented in earlier reports [5, 7]. For the purpose of this study, only patients who reported CTEPH symptoms or with known timing of symptoms onset were considered.

The aims of the current analysis were to 1) assess the impact of longer delay on prognosis, 2) describe the patterns of diagnostic delay and 3) evaluate predictors for longer delay.

Diagnostic delay was defined as the time of first self-reported onset of symptoms to the day of CTEPH diagnosis by right heart catheterisation, and was expressed in tertiles for the purposes of the present analysis. We partitioned total diagnostic delay into patient-associated delay, i.e. from the self-reported moment of symptom onset to first physician contact for these symptoms, and doctor-associated delay, defined as time from first physician contact to confirmation of CTEPH diagnosis.

Of all 679 patients included in the registry, no symptoms were recorded for 42 (6.2\%) patients who were all categorised as New York Heart Association (NYHA) class I. These 42 patients were excluded from further analysis. Median duration of follow-up was 37.7 months (interquartile range (IQR) 16.646.0 months). Overall, 321 (50\%) patients were female and mean age was 63 years; 77 (14\%) patients had cancer, 29 (4.8\%) had chronic heart disease, 131 (21\%) had concomitant conditions associated with pulmonary hypertension and $142(22 \%)$ were diagnosed with recurrent venous thromboembolism (VTE) during follow-up. Median patient-associated delay was 3.1 months (IQR 0-15 months) and median

@ERSpublications

This study identified CTEPH patient characteristics that were predictive of longer diagnostic delay, which in turn was associated with higher pulmonary artery pressures and an increased risk of death http://ow.ly/yCZx30m9TQS

Cite this article as: Klok FA, Barco S, Konstantinides SV, et al. Determinants of diagnostic delay in chronic thromboembolic pulmonary hypertension: results from the European CTEPH Registry. Eur Respir J 2018; 52: 1801687 [https://doi.org/10.1183/13993003.01687-2018]. 
doctor-associated delay was 5.4 months (IQR 2.6-13 months). We observed a nonlinear distribution of delays suggesting that longer patient-associated delay corresponded to shorter doctor-associated delay and vice versa.

Of all studied variables included in a penalised ordinal logistic regression model, recurrent VTE and obesity were selected for their association with a longer delay with odds ratios of 1.66 (95\% CI 1.16-2.38) and 1.52 (95\% CI 1.04-2.24), respectively. Vice versa, chest pain and fatigue were associated with a shorter delay, with odds ratios of 0.65 (95\% CI $0.44-0.97$ ) and 0.72 (95\% CI $0.53-0.98$ ), respectively. Age, sex and severity of degree of exertional dyspnoea (as expressed as NYHA classification) were not associated with longer or shorter delay. In one-way ANOVA, patients with a longer delay had less favourable pulmonary haemodynamics with mean pulmonary arterial pressure in the third tertile (diagnostic delay $>24.5$ months) being $\sim 4.5 \mathrm{mmHg}$ higher than in the second (diagnostic delay 9.8-24.5 months) or first tertile (diagnostic delay $<9.8$ months), respectively $(\mathrm{p}<0.001)$. Longer delay did not impact the chances of the patient to be judged operable (OR 1.08 (95\% CI 0.77-1.51), third tertile versus first and second tertile), the actual performance of PEA (OR 1.19 (95\% CI 0.85-1.66), third tertile versus first and second tertile) or surgical complications (OR 1.27 (95\% CI 0.88-1.84), third tertile versus first and second tertile). However, longer delay was associated with a higher risk of all-cause mortality (model 1 in table 1). In a multivariable Cox regression model, patients in the third tertile of delay were found to have an adjusted hazard ratio (HR) of 1.47 (95\% CI 0.99-2.15) compared to the first and second tertile together, with adjustment for age, sex, PEA and severity of symptoms. The same adjusted model comparing patients in the third tertile to the other two tertiles separately (model 2 in table 1) clarified that the largest difference in mortality risk was found between the third and first tertile (adjusted HR 1.60, 95\% CI 1.02-2.50), while there was no relevant difference between second and first tertile (adjusted HR 1.20, 95\% CI 0.75-1.92). The predicted probability of death grew exponentially if the diagnostic delay was accounted as a continuous variable regardless of whether patients underwent PEA or not: these observations underpin the lack of an interaction between diagnostic delay and the decision to perform PEA.

In this study, we identified subgroups of CTEPH patients with longer diagnostic delay, which was associated with less favourable haemodynamic profile and shorter survival, possibly reflecting more severe concomitant secondary vascular disease and poorer right ventricular function. As CPETH is a severe cardiovascular disease, it may come to no surprise that delay in diagnosis (and treatment initiation) of CTEPH has a negative impact on prognosis. However, the diagnostic process of CTEPH is complex and is influenced by many different patient- as well as doctor-driven factors [8-10]. Even for acute PE, diagnostic delay was found to have little prognostic significance in epidemiological studies for the same reasons $[11,12]$.

\begin{tabular}{lll} 
TABLE 1 Cox regression analysis for all-cause mortality & \\
& Unadjusted HR (95\% CI) & Adjusted HR (95\% CI) \\
\hline Model 1 & & \\
First and second delay tertiles & Ref. & Ref. \\
Third delay tertile & $1.34(0.92-1.96)$ & $1.47(0.999-2.15)$ \\
Age per unit increase & $1.03(1.01-1.04)$ & $1.01(0.997-1.03)$ \\
Male sex & Ref. & Ref. \\
Female sex & $0.74(0.51-1.07)$ & $0.60(0.41-0.87)$ \\
PEA not performed & Ref. & Ref. \\
PEA performed & $0.28(0.19-0.42)$ & $0.28(0.18-0.43)$ \\
NYHA I-II & Ref. & Ref. \\
NYHA III-IV & $2.46(1.29-4.71)$ & $2.21(1.15-4.24)$ \\
Model 2 & & \\
First delay tertile & Ref. & Ref. \\
Second delay tertile & $0.94(0.59-1.50)$ & $1.20(0.75-1.92)$ \\
Third delay tertile & $1.30(0.84-2.03)$ & $1.60(1.02-2.50)$ \\
Age per unit increase & $1.03(1.01-1.04)$ & $1.02(0.998-1.03)$ \\
Male sex & Ref. & Ref. \\
Female sex & $0.74(0.51-1.07)$ & $0.59(0.40-0.85)$ \\
PEA not performed & Ref. & Ref. \\
PEA performed & $0.28(0.19-0.42)$ & 0.28 (0.18-0.42) \\
NYHA I-II & Ref. & Ref. \\
NYHA III-IV & $2.46(1.29-4.71)$ & $2.25(1.17-4.33)$
\end{tabular}

HR: hazard ratio; PEA: pulmonary endarterectomy; NYHA: New York Heart Association. 
Recurrent VTE and obesity were found to be associated with longer diagnostic delay. For the former, it is likely that this diagnosis was incorrect, since the spectrum of CTEPH symptoms and findings on imaging tests may resemble that of recurrent acute PE $[13,14]$. In such cases of potential misclassification, while awaiting the results of (intensified) anticoagulant therapy, diagnostic tests for CTEPH were likely to be delayed. Consistently, physicians and patients may erroneously attribute nonspecific symptoms to obesity and refrain from ruling out underlying pathology such as CTEPH, especially in the absence of a prior VTE diagnosis. The opposite can be argued for chest pain, being the presentation of acute thoracic conditions that often prompt diagnostic evaluation that could show clues of pulmonary hypertension [15].

The main limitations of our study are 1) recall bias, 2) lack of standardisation of the reported episodes of acute PE and 3) absence of detailed information of the diagnostic reasoning of the treating physicians.

In conclusion, we have, for the first time, demonstrated that longer delay negatively impacts prognosis of CTEPH. Although our results should be regarded as hypothesis generating, they support the need for increased CTEPH awareness by physicians, particularly those commonly diagnosing and treating acute PE.

Frederikus A. Klok $\oplus^{1,2}$, Stefano Barco $\oplus^{1}$, Stavros V. Konstantinides ${ }^{1}$, Philippe Dartevelle ${ }^{3}$, Elie Fadel ${ }^{4}$, David Jenkins ${ }^{5}$, Nick H. Kim ${ }^{6}$, Michael Madani $^{7}$, Hiromi Matsubara ${ }^{8}$, Eckhard Mayer ${ }^{9}$, Joanna Pepke-Zaba ${ }^{10}$, Marion Delcroix $\oplus^{11}$ and Irene M. Lang ${ }^{12}$

${ }^{1}$ Center for Thrombosis and Hemostasis, University Medical Center of the Johannes Gutenberg University, Mainz, Germany. ${ }^{2}$ Dept of Medicine - Thrombosis and Hemostasis, Leiden University Medical Center, Leiden, The Netherlands. ${ }^{3}$ Dept of Thoracic and Vascular Surgery and Heart-Lung Transplantation, Hôpital Marie-Lannelongue, Paris-Sud Univ., Paris, France. ${ }^{4}$ Univ. Paris-Sud, Faculté de Médecine, Université Paris-Saclay, Le Kremlin-Bicêtre, France. ${ }^{5}$ Dept of Cardiothoracic Surgery, Papworth Hospital, Cambridge, UK. ${ }^{6}$ Division of Pulmonary and Critical Care Medicine, University of California San Diego, La Jolla, CA, USA. ${ }^{7}$ Division of Cardiovascular and Thoracic Surgery, University of California San Diego, La Jolla, CA, USA. ${ }^{8}$ Dept of Clinical Science, National Hospital Organization Okayama Medical Center, Okayama, Japan. ${ }^{9}$ Kerckhoff Heart and Lung Center, Bad Nauheim, Germany. ${ }^{10}$ Pulmonary Vascular Disease Unit, Papworth Hospital, Cambridge, UK. ${ }^{11}$ Dept of Pneumology, University Hospitals Leuven, Leuven, Belgium. ${ }^{12}$ Dept of Internal Medicine II, Division of Cardiology, Vienna General Hospital, Medical University of Vienna, Vienna, Austria.

Correspondence: Frederikus A. Klok, Dept of Thrombosis and Hemostasis, LUMC, Albinusdreef 2, 2300 RC Leiden, The Netherlands. E-mail: f.a.klok@lumc.nl

Received: Aug 052018 | Accepted after revision: Sept 232018

Author contributions: F.A. Klok contributed to the concept and design of the study, interpretation of the results, writing of the manuscript, and gave final approval. S. Barco contributed to the design of the study, statistical analysis, interpretation of the results, writing of the manuscript, and gave final approval. S.V. Konstantinides, M. Delcroix, and I.M. Lang contributed to the design of the study, interpretation of the results, critical revision of the manuscript for important intellectual content, and gave final approval. All other authors contributed to the design of the study and provided important intellectual content. All authors approved of the final manuscript. The authors are grateful to the steering committee and investigators of the European CTEPH Registry for being allowed to use the data for the present analysis.

Conflict of interest: F.A. Klok reports receiving research grants from Bayer, Bristol-Myers Squibb, Boehringer-Ingelheim, MSD, Daiichi-Sankyo, Actelion, the Dutch Thrombosis Association and the Dutch Heart Foundation. S. Barco reports having travel and congress costs covered by Bayer HealthCare and Daiichi Sankyo, outside the submitted work. S.V. Konstantinides has received consultancy and lecture honoraria from Bayer HealthCare, Boehringer Ingelheim, Daiichi-Sankyo, Pfizer and Bristol-Myers Squibb; payment for travel accommodation/meeting expenses from Bayer HealthCare; and institutional grants from Boehringer Ingelheim, Bayer HealthCare and Daiichi-Sankyo. P. Dartevelle has nothing to disclose. E. Fadel has nothing to disclose. D. Jenkins reports receiving honoraria for lectures on PEA and support for a PEA bridging trial from Bayer, and honoraria for work on the MERIT trial as an adjudicator from Actelion, outside the submitted work. N.H. Kim reports receiving research support form Actelion, Bayer and Merck, and has received lecture honoraria from Bayer. M. Madani has nothing to disclose. H. Matsubara reports receiving honoraria for lectures from Actelion Pharmaceuticals Japan, Ltd, AOP Orphan Pharmaceuticals AG, Bayer Yakuhin, Ltd, Nippon Shinyaku, Co., Ltd, Pfizer Japan, Inc., and Kaneka Medix Corporation, outside the submitted work. E. Mayer reports receiving speaker and consulting fees from Actelion, Bayer and MSD, and speaker fees from Pfizer, outside the submitted work. J. Pepke-Zaba or her institution have received research and educational grants from Actelion, Merck, and Bayer. She has also served on advisory boards for Actelion, J\&J, Merck, Bayer and GSK. M. Delcroix has been an investigator, speaker, consultant or steering committee member for Actelion, Bayer, Eli Lilly, GlaxoSmithKline, MSD and Pfizer; and has received research grants from Actelion. I.M. Lang reports receiving lecture fees from MSD, grants and lecture fees from AOPORphan Pharma, and grants and personal fees from Actelion, outside the submitted work.

Support statement: The work of F.A. Klok, S. Barco and S.V. Konstantinides is supported by the German Federal Ministry of Education and Research (BMBF 01EO1003 and 01EO1503).

\section{References}

1 Lang IM, Madani M. Update on chronic thromboembolic pulmonary hypertension. Circulation 2014; 130: 508-518.

2 Delcroix M, Kerr K, Fedullo P. Chronic thromboembolic pulmonary hypertension. epidemiology and risk factors. Ann Am Thorac Soc 2016; 13: Suppl. 3, S201-S206. 
3 Simonneau G, Torbicki A, Dorfmuller P, et al. The pathophysiology of chronic thromboembolic pulmonary hypertension. Eur Respir Rev 2017; 26: 160112.

4 Ende-Verhaar YM, Cannegieter SC, Vonk Noordegraaf A, et al. Incidence of chronic thromboembolic pulmonary hypertension after acute pulmonary embolism: a contemporary view of the published literature. Eur Respir J 2017; 49: 1601792.

5 Delcroix M, Lang I, Pepke-Zaba J, et al. Long-term outcome of patients with chronic thromboembolic pulmonary hypertension: results from an international prospective registry. Circulation 2016; 133: 859-871.

6 Jenkins D, Madani M, Fadel E, et al. Pulmonary endarterectomy in the management of chronic thromboembolic pulmonary hypertension. Eur Respir Rev 2017; 26: 160111.

7 Pepke-Zaba J, Delcroix M, Lang I, et al. Chronic thromboembolic pulmonary hypertension (CTEPH): results from an international prospective registry. Circulation 2011; 124: 1973-1981.

8 Gall H, Preston IR, Hinzmann B, et al. An international physician survey of chronic thromboembolic pulmonary hypertension management. Pulm Circ 2016; 6: 472-482.

9 Ende-Verhaar YM, van den Hout WB, Bogaard HJ, et al. Healthcare utilization in chronic thromboembolic pulmonary hypertension after acute pulmonary embolism. J Thromb Haemost 2018; 16: 2168-2174.

10 Ende-Verhaar YM, Huisman MV, Klok FA. To screen or not to screen for chronic thromboembolic pulmonary hypertension after acute pulmonary embolism. Thromb Res 2017; 151: 1-7.

11 den Exter PL, van Es J, Erkens PM, et al. Impact of delay in clinical presentation on the diagnostic management and prognosis of patients with suspected pulmonary embolism. Am J Respir Crit Care Med 2013; 187: 1369-1373.

12 Pasha SM, Klok FA, van der Bijl N, et al. Right ventricular function and thrombus load in patients with pulmonary embolism and diagnostic delay. J Thromb Haemost 2014; 12: 172-176.

13 Guerin L, Couturaud F, Parent F, et al. Prevalence of chronic thromboembolic pulmonary hypertension after acute pulmonary embolism. Prevalence of CTEPH after pulmonary embolism. Thromb Haemost 2014; 112: 598-605.

14 Klok FA, Delcroix M, Bogaard HJ. Chronic thromboembolic pulmonary hypertension from the perspective of patients with pulmonary embolism. J Thromb Haemost 2018; 16: 1040-1051.

15 Galie N, Humbert M, Vachiery JL, et al. 2015 ESC/ERS Guidelines for the diagnosis and treatment of pulmonary hypertension. Eur Heart J 2016; 37: 67-119. 